\title{
Efficacy of Standardised Treatments Combined with Ubenimex in Patients with Malignant Tumors
}

\author{
Xin Hu, Haofeng Xiong, Shiying Huang, Ting Mao, Liudi Yang and Tong Su \\ Department of Oral and Maxillofacial Surgery, Xiangya Hospital, Central South University, China
}

\begin{abstract}
Ubenimex is widely used as an immunomodulator in the treatment of leukemia and non-small cell lung cancer to improve the anti-tumor treatment effect. However, there has not been any multicenter randomised controlled trials to study its impact on the prognosis of cancer patients. The authors aimed to conduct a meta-analysis to initially study these issues. Pubmed, Cochrane Library and EMbase were searched. Randomised controlled trials of the effects of ubenimex on the survival rate of malignant tumor patients were included in the meta-analysis. Survival rate ratio (OR) and $95 \%$ confidence interval $(95 \% \mathrm{Cl})$ between two groups were used to evaluate the efficacy of ubenimex. Fixed effects models were used for meta-analysis. A total of 1,372 cases (684 in the ubenimex group and 688 in the control group) of five studies were included. Between the ubenimex group and the control group, the 1-year OR was $1.40(95 \% \mathrm{Cl}=1.06$ to 1.85$)$, the 2 -year OR was $1.43(95 \% \mathrm{Cl}=1.08$ to 1.89$)$ and the 3 -year OR was $1.39(95 \% \mathrm{Cl}=1.07$ to 1.81$)$. Standardised treatments combined with ubenimex may improve the survival rate of patients with malignant tumors.
\end{abstract}

Key Words: Malignant tumors, Ubenimex, Randomised controlled trials, Meta-analysis.

How to cite this article: Hu X, Xiong H, Huang S, Mao T, Yang L, Su T. Efficacy of Standardised Treatments Combined with Ubenimex in Patients with Malignant Tumors. J Coll Physicians Surg Pak 2021; 31(02):206-209.

\section{INTRODUCTION}

Malignant tumors are important and serious health concern. In the United States, approximately 4,600 people are diagnosed with malignant tumors each day, and 1,650 people die of malignant tumors each day. ${ }^{1}$ After a series of standardised treatments (surgery, chemotherapy, radiotherapy etc.), the recurrence rates of many kinds of malignant tumors remain high. In addition to those treatments, combined with some low-toxic adjunctive agents, to improve patient survival, has also become the goals of many scholars and researchers.

The immune system plays an important role in the body's own anti-tumor activities, and immunotherapy have been increasingly important in the treatment of malignant tumors. ${ }^{2,3}$ In many malignant tumors fields, patients with a wide degree of $\mathrm{CD} 8^{+} \mathrm{T}$ cell infiltration are indicated to have a better prognosis. ${ }^{4,5}$ The number of natural killer (NK) cells was positively correlated with the prognosis of liver cancer. ${ }^{6,7}$ Improving the function of these immune cells, may become a new way to improve the survival rate of malignant tumors.

Correspondence to: Dr. Tong Su, Department of Oral and Maxillofacial Surgery, Xiangya Hospital, Central South University, China

E-mail: sutong@csu.edu.cn

Received: October 24, 2019; Revised: September 27, 2020;

Accepted: December 28, 2020

DOI: https://doi.org/10.29271/jcpsp.2021.02.206
Ubenimex is a CD13 inhibitor that enhances immunity by enhancing T cells' viability and enhancing NK cells' lethality. ${ }^{8}$ Ubenimex can also directly exert its anti-cancer effect by interfering with tumor cell metabolism. ${ }^{9}$ At present, ubenimex is widely used as an adjuvant drug in the treatment of leukemia and non-small cell lung cancer to improve the effects of anti-tumor treatment. ${ }^{10,11}$ There has been no report about any serious side effects of ubenimex. However, whether patients with malignant tumors need to use ubenimex is still controversial, and there are still no multicentre large randomised controlled trials to investigate the relationship between ubenimex and prognosis of patients with malignant tumors. This meta-analysis was aimed to initially study these issues, and provide decision-making reference for clinical practice.

\section{METHODOLOGY}

Research retrieval adopted the theme words-joint-free words retrieval method. Search terms included bestatin, ubenimex, (D-Leu)-(R-( $\left.\left.\mathrm{R}^{*}, \mathrm{~S}^{*}\right)\right)$-isomer, tumour, tumor, malignant neoplasm, cancer, randomised controlled trial (RCT). Pubmed, Cochrane library, and EMbase were searched to collect RCTs about the effects of ubenimex on the survival rate of malignant tumor patients from the establishment of the databases to January 2019.

The inclusion criteria were a randomised controlled trial (RCT); participants must be diagnosed as malignant tumor patients pathologically, regardless of the type of tumor;

the control group consisted of patients who received only stan- 
dardised treatment, and the ubenimex group was consisted of patients who received standardised treatment and combined with ubenimex, regardless of the dose and frequency; recurrence time and the number of patients who had a recurrence are outcome measures, were available. The exclusion criteria were studies, where clinical prognostic indicators did not include information on patient survival, or survival curves, insufficient data, extremely small sample size, or too many cases lost-to-follow-up, where literary responsibility were unknown, or where full texts were not available, were excluded.

Two researchers independently read, selected the studies, and extracted the data separately. Whether to include divergent studies were decided through consultation. When opinions were not met, a third researcher reviewed and decided. The extracted data included the title, author, publication time, region, trial control, time to relapse, and number of cases. All results had been evaluated by subject experts. A total of 109 records were identified through Pubmed, Cochrane Library and EMbase searching. After removing duplicated records, 53 records left. According to the title of the article, 13 studies which obviously did not meet the inclusion criteria, were excluded; and 40 records were left. After full-text articles assessed, 34 recordswere excluded, and 5 studieswere included in the meta-analysis. Cochrane risk bias evaluation tool was used to assess the bias risk by the rules of Cochrane Handbook. ${ }^{12}$

RevMan 5.3 was used in this meta-analysis. Survival rate ratio (OR) and 95\% confidence interval $(95 \% \mathrm{Cl})$ between the two groups were used to evaluate the efficacy of ubenimex. Chisquare test was used to investigate the heterogeneity among the five studies. If the heterogeneity between the studies was small ( $p \geq 0.1, l^{2} \leq 50 \%$ ), the fixed effect model was used to analyse the data. If the heterogeneity among the studies was large $\left(p \leq 0.1, I^{2} \geq 50 \%\right)$, after eliminating human factors such as statistical errors, a random effect model was used to analyse the data. If there was a significant clinical heterogeneity in each study, subgroup analysis or sensitivity analysis was used. Statistical significance was defined as $p<0.05$.

\section{RESULTS}

A total of 109 articles were retrieved. After screening, five RCTs $(n=1372)$ met the criteria and were finally included in the study. ${ }^{10,13-16}$ Basic characteristics of included studies were showed in Table I. Bias risk assessment results were showed in Figure1, showing the type of bias and the total extent of the five studies included. Green, yellow, and red represent low risk, unclear risk, and high risk. The proportion of the color represents the size of the bias. Heterogeneity test results are shown in Figure 2-4.

Figure 2 shows the result of meta-analysis for 1-year survival rate. five studies were included. ${ }^{10,13,16}$ Fixed effect model was used to analyse the data. There was no difference in 1-year survival rate between the ubenimex group and the control group. The OR was $1.40(95 \% \mathrm{Cl}=1.06$ to 1.85$)$.

Figure 3 shows the result of meta-analysis for 2-year survival rate. five studies were included. ${ }^{10,13-16}$ Fixed effect model was used to analyse the data. 2-year survival rate of the ubenimex group was significantly higher than that of the control group; the OR was $1.43(95 \% \mathrm{Cl}=1.08$ to 1.89$)$.

Figure 4 shows the result of meta-analysis for 3-year survival rate. Five studies were included. ${ }^{10,13-16}$ Fixed effect model was used to analyse the data. 3-year survival rate of the ubenimex group was significantly higher than that of the control group; the OR was $1.39(95 \% \mathrm{Cl}=1.07$ to 1.81$)$.

\section{DISCUSSION}

The intractability of malignant tumors is a worldwide problem. Although there are many kinds of treatments for malignant tumors; still, no malignant tumor could be claimed to be cured completely. Any method that can improve the survival rate of cancer patients is worth this effort. In the previous study, it was mentioned that metformin significantly improved the prognosis of oral cancer patients with type 2 diabetes mellitus. ${ }^{17}$ At the same time, ubenimex, as a low toxicity immunomodulator and as an anticancer drug, has been used in clinic for decades. In invitro experiment, ubenimex induces the autophagic cell death in prostate cancer cells and renal cancer cells. ${ }^{18,19}$ It can also enhance the effects of anticancer drugs in hepatocellular cancer. ${ }^{20}$ Compared to in-vitro experiments, there are only a few clinical trials on the anti-tumor effect of ubenimex. In the routinetreatment of malignanttumors, whether the use of ubenimex can improve the survival rate of patients, becomes a clinical problem to be solved.

This meta-analysis included a total of five RCT studies. The results (Figure 3 and Figure 4 ) show that ubenimex may improve the 1-year, 2-year, and 3-year-survival rates of patients with malignant tumors. With the development of high-throughput sequencing technology, human research on cancer has entered the information age. Tumour-infiltrating immune cell has been found playing an increasing important role regarding the anti- or pro-tumorigenesis and prognosis of tumor patients. The immune checkpoint blockade and chimeric antigen receptor T-cell therapies have achieved amazing success. $^{21}$ Yamazaki et al. found that natural killer and lymphokine activated killer cell activity of patients with hematological malignancies was significantly lower than normal people, and ubenimex administration can not only elevate the activity of natural killer and lymphokine activated killer cell, but also increase the absolute numbers of helper T-cells, cytotoxic T-cells and natural killer cells. ${ }^{22}$ Ubenimex treatment could enhance the susceptibility of gastric cancer cell to lymphokineactivated killer cells. ${ }^{23}$ Natural killer cell, B-cell, CD8 ${ }^{+} \mathrm{T}$-cell, and active $\mathrm{CD} 4^{+}$memory $\mathrm{T}$-cell were found to be survival-favourable immune cells in breast cancer. ${ }^{24}$ Thus, ubenimex may improve the tumor immune microenvironment to enhance the clinical therapeutic effect on tumor. Ubenimex may improve the survival rate of patients with malignant tumors by improving the immune system to eliminate more dormant malignant tumorcells. 
Table I: Basic characteristics of included studies.

\begin{tabular}{|c|c|c|c|c|c|c|}
\hline \multirow{2}{*}{ Authors } & \multirow{2}{*}{$\begin{array}{l}\text { Published } \\
\text { date }\end{array}$} & \multirow{2}{*}{ Country } & \multirow{2}{*}{ Diagnosis of objects } & \multirow{2}{*}{ Standardised treatments } & \multicolumn{2}{|c|}{ Treatment } \\
\hline & & & & & Ubenimex group & Control group \\
\hline C. Mouritzen ${ }^{13}$ & 1990 & Denmark & $\begin{array}{c}\text { Stage I and stage II non-small cell } \\
\text { lung cancer }\end{array}$ & Surgery & Ubenimex & Blank \\
\hline Henric Blomgren $^{14}$ & 1987 & Sweden & $\begin{array}{c}\text { Transitional cell carcinoma of the } \\
\text { bladder }\end{array}$ & Radiotherapy & Ubenimex & Blank \\
\hline M.Takada ${ }^{15}$ & 1990 & Japan & Inoperable lung cancer & $\begin{array}{l}\text { Chemotherapy or } \\
\text { radiotherapy }\end{array}$ & Ubenimex & Blank \\
\hline T.Yasumitsu ${ }^{10}$ & 1990 & Japan & Resected lung cancer & Surgery & Ubenimex & Blank \\
\hline Yukito Ichinose ${ }^{16}$ & 2003 & Japan & $\begin{array}{c}\text { Resected state I squamous-cell lung } \\
\text { carcinoma }\end{array}$ & Surgery & Ubenimex & Placebo \\
\hline
\end{tabular}

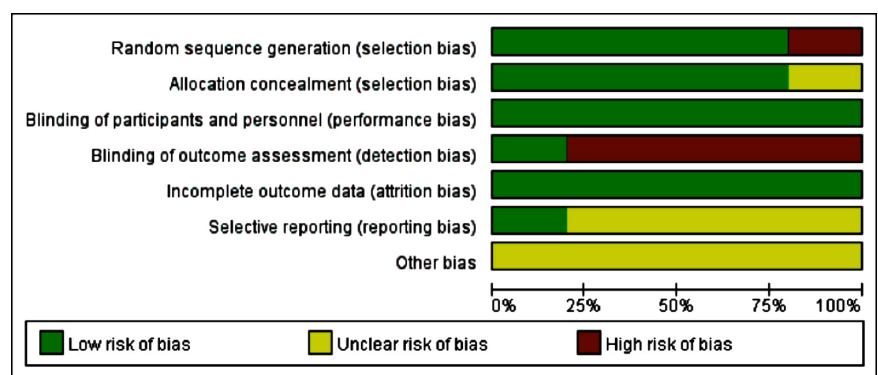

Figure 1: Bias risk assessment results. ${ }^{10,13}$

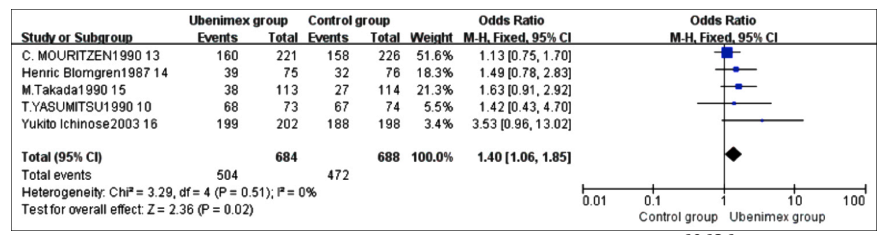

Figure 2: Meta-analysis results of 1-year survival rate. ${ }^{10,13-1}$

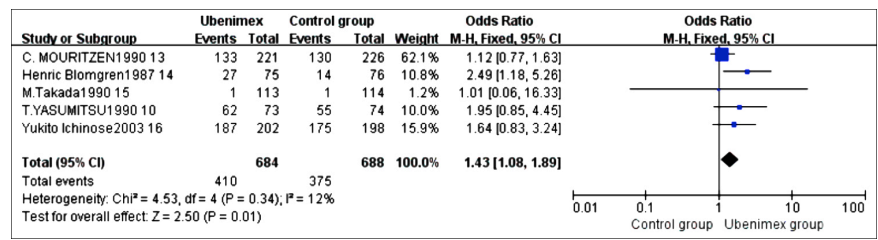

Figure 3: Meta-analysis results of 2-year survival rate. ${ }^{10,13-16}$

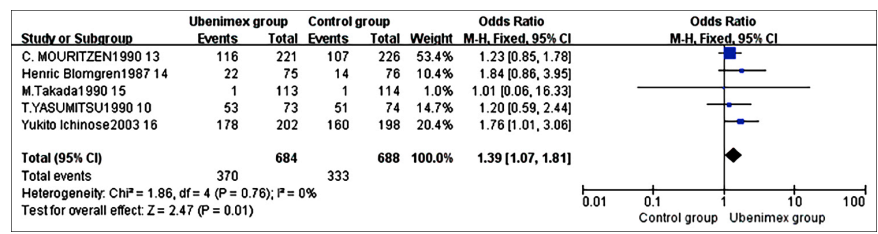

Figure 4: Meta-analysis results of 3-year survival rate. ${ }^{10,13-16}$

Besides the impacts on immune cells, ubenimex has been found to have many direct anticancer effects. Wang et al. found that ubenimex might be a perfect adjunctive therapy for melanoma, when combined with Akt inhibitor. ${ }^{25}$ Ubenimex could also induce apoptotic and autophagic cell death through the ROS/ERK pathway. ${ }^{26}$ Inoi et al. found that ubenimex played am important role im inhibiting the growth of human choriocarcinoma in nude mice by its direct cytostatic activity. ${ }^{27}$

Of course, there were no subgroup analyses of different tumor types in this study because of the limited number of studies. It is not yet possible to determine the effect of ubenimex on the survival rate of patients with specific tumor types. The results of this study are still to be further studied and analysed.
Survival was the most concerned outcome of the included studies. In the forest plots of Figures 2-4, the results of the five studies were consistent. No significant heterogeneity was found after statistical analysis. However, the limitations of this study still existed including the following aspects: Firstly, as shown in Figure 1, only one selected RCT was blinding of outcome assessment, so detection bias is inevitable. Based on available information, there is no way to judge whether the authors have reported the results selectively; so there may be a certain degree of reporting bias. Secondly, the tumor types are different, and the prognosis of different tumors is different. Thirdly, although the included studies were all RCT studies, the overall number of studies was small, which might cause some bias in the results. Fourthly, despite extensive searches, publication bias cannot be ruled out, such as some grey or missing studies in conference. Finally, the areas and occupations of the patients included in the studies are different, so there may be an inevitable bias in the research results.

\section{CONCLUSION}

Ubenimex may improve the survival rate of cancer patients. Clinicians can use ubenimex to improve survival rate based on routine treatment. However, due to the above limitations of the study, the accuracy of the results may be affected. The results also require multiple centres, large numbers of samples, and long-term follow-up randomised double-blind controlled trials to confirm the results.

\section{FUNDING:}

This work was supported by the National Natural Science Foundation (81873717); the Natural Science Foundation of Hunan Province (2019JJ40508); and the Graduate Students Independently Explore Innovative Projects of Central South University (2019zzts787), China.

\section{CONFLICT OF INTEREST:}

The authors declared no conflict of interest.

\section{AUTHORS' CONTRIBUTION:}

$\mathrm{XH}$ : Substantial contributions to the conception and design of the work.

$\mathrm{HX}$ : Acquisition, analysis of data for the work.

$\mathrm{SH}$ : Drafting the work.

TM: Analysis of data. 
LY: Acquisition of data.

TS: Final approval of the version to be published; and agreed to be accountable in all respects of the work.

\section{REFERENCES}

1. Siegel RL, Miller KD, Jemal A. Cancer statistics, 2017. CA Cancer J Clin 2017; 67(1):7-30. doi: 10.3322/caac.21387.

2. Pardoll DM. The blockade of immune checkpoints in cancer immunotherapy. Nature Reviews Cancer 2012; 12(4): 252-64. doi: 10.1038/nrc3239.

3. Drake CG, Lipson EJ, Brahmer JR. Breathing new life into immunotherapy: Review of melanoma, lung and kidney cancer. Nature Reviews Clinical Oncology 2014; 11(1): 24-37. doi: 10.1038/nrclinonc.2013.208.

4. Yiping Y. Cancer immunotherapy: Harnessing the immune system to battle cancer. J Clinical Investigation 2015; 125(9):3335-7. doi: 10.1172/JCl83871.

5. Galon J, Costes A, Sanchez-Cabo F, Kirilovsky A, Mlecnik B, Lagorce-Pagès, $C$, et al. Type, density, and location of immune cells within human colorectal tumors predict clinical outcome. Science 2006; 313(5795):1960-4. doi: 10.1126/science. 1129139.

6. Chew V, Chen J, Lee D, Loh E, Lee J, Lim KH, et al. Chemokine-driven lymphocyte infiltration: An early intra-tumoural event determining long-term survival in resectable hepatocellular carcinoma. Gut 2012; 61(3):427-38. doi: 10.1136/gutjnI-2011-300509.

7. Bastian H, Torsten V, Lars O, Jaba G, Fei Z, Heiner W, et al. Myeloid derived suppressor cells inhibit natural killer cells in patients with hepatocellular carcinoma via the NKp30 receptor. Hepatol 2010; 50(3):799-807. doi: 10.1002/ hep.23054.

8. Suda H, Aoyagi T, Takeuchi T, Umezawa H. Inhibition of aminopeptidase $B$ and leucine aminopeptidase by bestatin and its stereoisomer. Archives Biochemistry Biophysics 1976; 177(1):196-200. doi: 10.1016/0003-9861(76)90429-x.

9. Constam DB, Tobler AR, Rensing-Ehl A, Kemler I, Hersh LB, Fontana A. Puromycin-sensitive aminopeptidase. Sequence analysis, expression, and functional characterisation. J Biological Chemistry 1995; 270(45):26931-9. doi: 10.1074/ jbc. 270.45.26931.

10. Yasumitsu T, Ohshima S, Nakano N, Kotake $Y$, Tominaga S. Bestatin in resected lung cancer. A randomised clinical trial. Acta Oncologica 1990; 29(6):827-31. doi: 10.3109/0284186 9009093009.

11. Review of ubenimex (Bestatin): Clinical research. Biomedicine and Pharmacotherapy.

12. Higgins JP, Green S. Cochrane Handbook for Systematic Reviews of Interventions: Cochrane Book Series2008.

13. Mouritzen C. Bestatin as adjuvant treatment in operated stage I and stage II non-small cell lung cancer. European lung cancer study group. Acta oncologica 1990; 29(6): 817-20. doi: 10.3109/02841869009093007.

14. Blomgren H, Naslund I, Esposti PL, Johansen L, Aaskoven O. Adjuvant Bestatin immunotherapy in patients with transitional cell carcinoma of the bladder. Clinical results of a randomised trial. Cancer immunology, immunotherapy 1987; 25(1):41-6. doi: 10.1007/BF00199299.

15. Takada M, Fukuoka M, Negoro S, Kusunoki Y, Matsui K, Masuda $\mathrm{N}$, et al. Combination therapy with bestatin in inoperable lung cancer. A randomised trial. Acta oncologica 1990; 29(6):82-5. doi: 10.3109/0284186900909.

16. Ichinose Y, Genka K, Koike T, Kato H, Watanabe Y, Mori T, et al. Randomised double-blind placebo-controlled trial of bestatin in patients with resected state I squamous-cell lung carcinoma. J National Cancer Institute 2003; 95(8): 605-10. doi: 10.1093/jnci/95.8.605.

17. Hu X, Xiong $\mathrm{H}$, Chen $\mathrm{W}$, Huang $\mathrm{L}$, Mao $\mathrm{T}$, Yang $\mathrm{L}$, et al. Metformin reduces the increased risk of oral squamous cell carcinoma recurrence in patients with type 2 diabetes mellitus: A cohort study with propensity score analyses. Surg Oncol 2020; 35:453-9. doi: 10.1016/j.suronc. 2020.09.023.

18. Wang $X$, Niu Z, Jia Y, Cui M, Han L, Zhang Y, et al. Ubenimex inhibits cell proliferation, migration and invasion by inhibiting the expression of APN and inducing autophagic cell death in prostate cancer cells. Oncology Report 2016; 35(4):2121-30. doi: 10.3892/or.2016.4611.

19. Liu S, Xie F, Wang H, Liu Z, Liu X, Sun L, et al. Ubenimex inhibits cell proliferation, migration and invasion in renal cell carcinoma: The effect is autophagy-associated. Oncology Reports 2015; 33(3):1372-80. doi: 10.3892/or. 2014.3693.

20. Yamashita M, Wada $H$, Eguchi $H$, Ogawa $H$, Yamada D, Noda $T$, et al. A CD13 inhibitor, ubenimex, synergistically enhances the effects of anticancer drugs in hepatocellular carcinoma. Int J Oncol 2016; 49(1):89-98. doi: 10.3892 ijo.2016.3496.

21. Yang Y. Cancer immunotherapy: Harnessing the immune system to battle cancer. J Clin Invest 2015; 125(9):3335-7. doi: 10.1172/JCl83871.

22. Yamazaki T, Sugiyama K, Ichihara K. Effect of ubenimex on the immune system of patients with hematological malignancies. Biomed Pharmacother 1991; 45(2-3):105-12. doi: 10.1016/ 0753-3322(91)90129-h.

23. Iwahashi M, Tanimura $H$, Yamaue $H$, Tani M, Noguchi K, Mizobata $\mathrm{S}$, et al. Ubenimex treatment enhances the susceptibility of gastric cancer cell lines to lymphokine-activated killer cells. Anticancer Res 1994; 14(4A):1563-8.

24. Huang CP, Liu LX, Shyr CR. Tumor-associated macrophages facilitate bladder cancer progression by increasing cell growth, migration, invasion and cytokine expression. Anticancer Res 2020; 40(5):2715-24. doi: 10.21873/ anticanres.14243.

25. Wang X, Liu Y, Wu R, Guo F, Zhang L, Cui M, et al. Role of ubenimex as an anticancer drug and its synergistic effect with Akt inhibitor in human A375 and A2058 cells. Onco Targets Ther 2018; 11:943-53. doi: 10.2147/OTT.S157480.

26. Wang $Y$, Pang B, Zhang R, Fu Y, Pang Q. Ubenimex induces apoptotic and autophagic cell death in rat GH3 and MMQ cells through the ROS/ERK pathway. Drug Des Devel Ther 2019; 13:3217-28. doi: 10.2147/DDDT.S218371.

27. Inoi K, Goto S, Nomura S, Isobe K, Nawa A, Okamoto T, et al. Aminopeptidase inhibitor ubenimex (bestatin) inhibits the growth of human choriocarcinoma in nude mice through its direct cytostatic activity. Anticancer Res 1995; 15(5B): 2081-7. 